\title{
Traveling Wave Deflector Design for Femtosecond Streak Camera
}

\author{
Chengquan Pei ${ }^{1}$ Shengli $\mathrm{Wu}^{1}$ Duan Luo ${ }^{2,3}$ Wenlong Wen ${ }^{2}$ Junkai Xu ${ }^{2,3}$ Jinshou Tian ${ }^{2,5 *}$ \\ Minrui Zhang ${ }^{2,3}$ Pin Chen ${ }^{2,3}$ Jianzhong Chen ${ }^{1}$ Rong Liu ${ }^{4}$ \\ (1. Key Laboratory for Physical Electronics and Devices of the Ministry of Education, Xi'an Jiaotong \\ University Xi'an 710049, China \\ 2. Xi'an Institute of Optics and Precision Mechanics, Chinese Academy of Sciences, Xi'an 710119, China \\ 3. University of Chinese Academy of Sciences, Beijing 100049, China \\ 4. Xi`an Technological University, Xi'an 710021, China \\ 5. Collaborative Innovation Center of Extreme Optics, Shanxi University, Taiyuan, Shanxi 030006, People's \\ Republic of China)
}

\begin{abstract}
In this paper, a traveling wave deflection deflector (TWD) with a slow-wave property induced by a microstrip transmission line is proposed for femtosecond streak cameras. The pass width and dispersion properties were simulated. In addition, the dynamic temporal resolution of the femtosecond camera was simulated by CST software. The results showed that with the proposed TWD a femtosecond streak camera can achieve a dynamic temporal resolution of less than $600 \mathrm{fs}$. Experiments were done to test the femtosecond streak camera, and an $800 \mathrm{fs}$ dynamic temporal resolution was obtained. Guidance is provided for optimizing a femtosecond streak camera to obtain higher temporal resolution.
\end{abstract}

Key words: Femtosecond streak camera, traveling wave deflection deflector, microstrip transmission line, dynamic temporal resolution

\section{Introduction}

Streak cameras (SCs) can convert the time variation of an ultrafast signal to a spatial variation and achieve picoseconds or even femtosecond measurements with high spatial resolution. They are widely used as ultrafast diagnostic tools in the physics of inertial confinement fusion (ICF) [1, 2], high-energy physics, and even biomedicine. However, to meet the demands of sophisticated diagnosis technology, a femtosecond SC should be developed. Research on SCs has been done extensively in 21 centers. In 2004, Zenghu Chang designed an SC with a $280 \mathrm{fs}$ temporal resolution [3]. A femtosecond SC with a $401 \mathrm{p} / \mathrm{mm}$ spatial resolution has been successfully designed by Russian scientists [4]. SCs have also been studied by Japanese scientists [5]; the dynamic range was up to 10,000:1. Moreover, neutron and ion SCs have also been developed successfully. However, the femtosecond SC for ultraviolet light was rare. An SC has a single scanning mode and a synchronous scanning mode. The synchronous scanning mode superposes a weak signal onto a fluorescent screen precisely by repetitive high-frequency synchronous scanning to improve detection sensitivity and dynamic range.

A femtosecond SC usually operates in synchronous scanning mode. The slit width of the SC is only 5 to $10 \mu \mathrm{m}$, to enable the deflection sensitivity and slope of the scanning voltage to be high enough. However, the higher the scanning voltage slope is, the wider the frequency bandwidth. In addition, the phase velocity and group velocity should match the electron 
velocity to achieve high deflection sensitivity. To enable this, we propose a traveling wave deflector (TWD) with a slow-wave property. A slow-wave structure [6] is a system that exchanges energy reciprocally between an electron beam and an electromagnetic wave. A TWD based on this slow-wave structure can match the electron velocity with the group velocity of a scanning signal applied to the 2 plates. This transfers the energy from the signal wave to the electrons, and the electrons achieve effective deflection. The main parameters of a TWD are deflection sensitivity, passband, and dispersion. However, research on TWD design is rare. Only in [7] were the stationary potential distribution and scattering parameter simulated by MAFIA software.

In this study, we designed a TWD based on a microstrip meander line with a slow-wave property for a femtosecond SC. The scattering parameter, phase velocity, and group velocity were simulated by CST software (Computer Simulation Technology). In addition, dynamic temporal resolution was also simulated. We ran an experiment to test dynamic temporal resolution; the result shows that the femtosecond SC can achieve a temporal resolution of $700 \mathrm{fs}$. This paper can provide guidance to design a TWD in theory, or a way to optimize a femtosecond SC to obtain higher temporal resolution.

\section{TWD design}

A femtosecond camera consists of a cathode, a grid, a pair of traveling wave plates, a magnetic lens, and a fluorescent screen, as shown in Fig. 1.

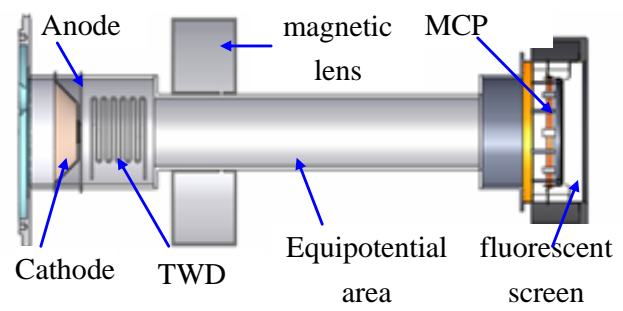

Fig. 1. Architecture of a femtosecond SC

The operating principle of a femtosecond SC can be described as follows: Photoelectrons emitted from a photocathode are accelerated by a grid. Then the electrons are deflected by a traveling wave plate; in this process, time variance information is transformed into spatial information. Finally, the electrons are focused by a magnetic lens and imaged on a charge coupling device (CCD). The TWD is essential for achieving femtosecond resolution. On the one hand, the electron velocity should match the group velocity of the TWD to obtain effective deflection. On the other hand, the TWD should have enough pass width to reduce the reflection wave. In this paper, a TWD in the form of a meander line, (Fig. 2a) is proposed. The main parameters of this TWD are gap (G), width (W), and length (L).

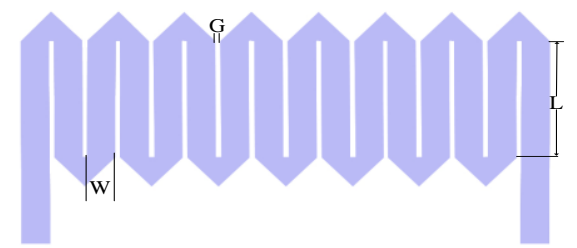

(a)

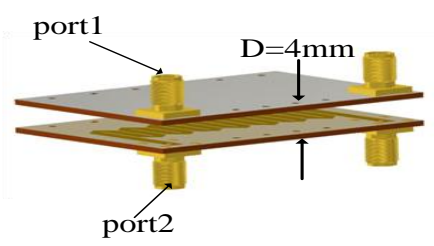

(b)

Fig. 2. Proposed TWD illustrated. (a) Meander line configuration. (b) Microstrip base. 
The TWD was designed based on the structure of microstrip transmission line. The purpose of the meander line was to slow the electrical deflecting pulse to match the beam velocity by having the electromagnetic field take a winding path. Figure $2 b$ shows the TWD used in a femtosecond SC that has 2 ports to import the scanning signals. The signals can be imported from port1, port2, or both ports. First, the meander line guides the electromagnetic field propagation transversely, whereas the electrons travel longitudinally. The velocity of the electrons is much slower than that of the scanning signal, so the electrons slip on the pulse for each leg of the meander line. The main characteristic of the proposed TWD are its bandwidth and dispersion.

\section{A. TWD Passband}

To analyze the pass width of the proposed TWD, the scatter parameter $\mathrm{S}_{12}$ was simulated for different gaps, widths, and lengths.

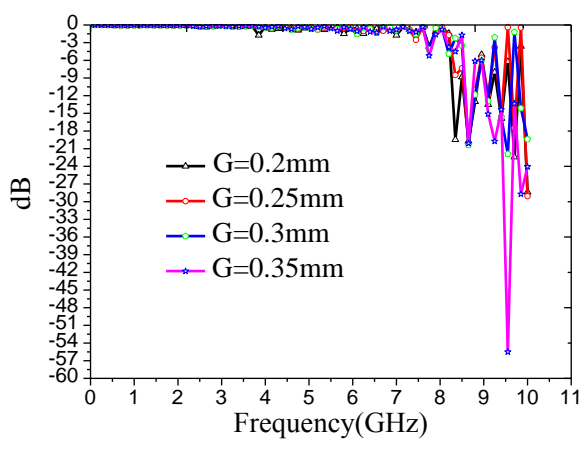

(a)

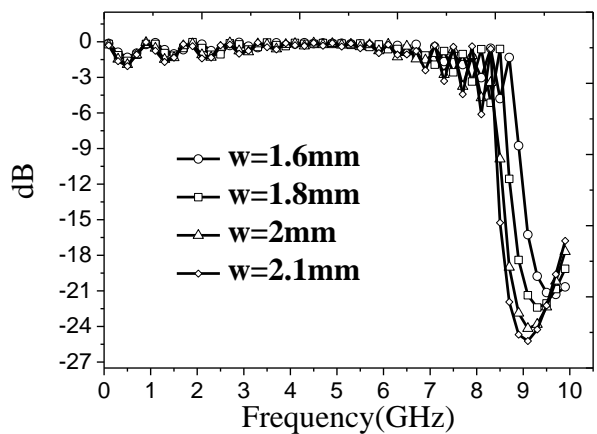

(c)

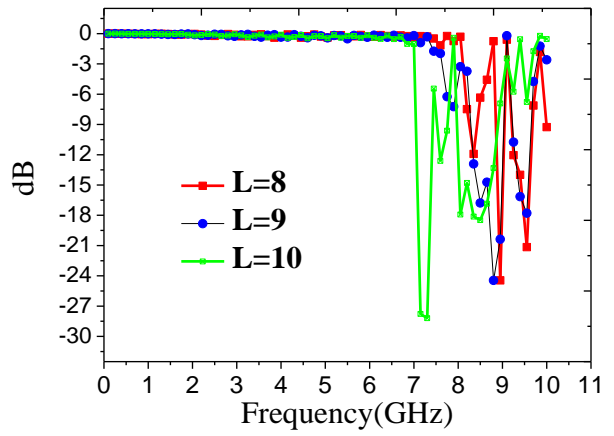

(b)

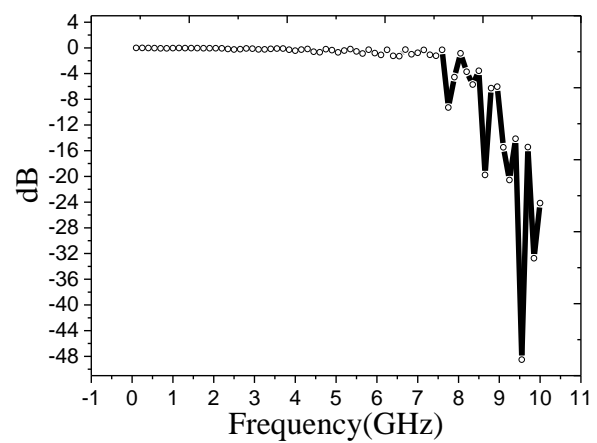

(d)

Fig. 3. Proposed TWD at different gaps, widths, and lengths. (a) Different gaps when $\mathrm{L}=8 \mathrm{~mm}$, $\mathrm{W}=2 \mathrm{~mm}$. (b) Different lengths when $\mathrm{G}=0.2, \mathrm{~W}=2 \mathrm{~mm}$. (c) Different widths when $\mathrm{L}=8 \mathrm{~mm}, \mathrm{G}=0.2 \mathrm{~mm}$. (d) $-3 \mathrm{~dB}$ passband of proposed TWD.

From Fig. 3 we can conclude that the greater the meander line width, the higher the achieved pass width; the smaller the gap, the lower the pass width; and the greater the length, the lower the pass width. However, the length should not less than the width of the cathode slit, and if the gap is larger, dispersibility of the electric field would be induced. Additionally, the TWD must have insufficient pass width to reduce the reflection waves. Figure $3 \mathrm{~d}$ shows the pass width of the TWD adopted in a femtosecond camera; the $-3 \mathrm{~dB}$ pass width was up to 7.5 
GHz.

\section{B. TWD Dispersion}

Because of the dispersion of the slow-wave structure, the phase velocity and group velocity of this TWD should be analyzed to select the property of the high voltage applied on the cathode. The propagation constant $k$ was simulated by CST software as shown in Fig. 5a, and then the phase velocity $v_{p}$ and group velocity $v_{g}$ can be derived by $v_{p}=2 \pi f / k$, $\frac{v_{p}}{v_{g}}=1-\frac{f}{v_{p}} \bullet \frac{d v_{p}}{d f}$ with the result shown in Fig. $5 \mathrm{~b}$.

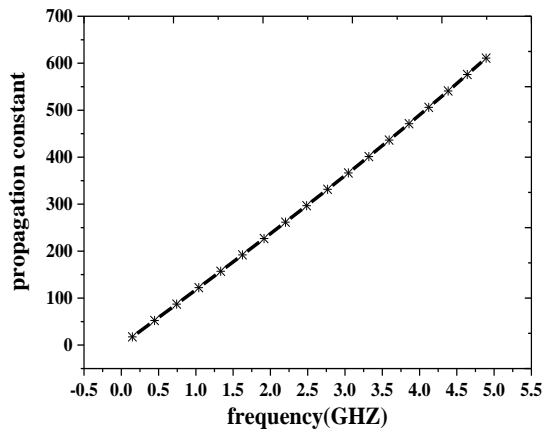

(a)

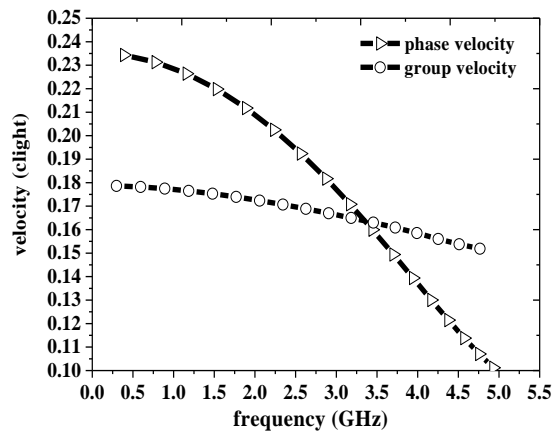

(b)

Fig. 5. Dispersion properties. (a) Propagation constant. (b) Phase velocity and group velocity.

A strong electric field was generated because of high voltage between the cathode and grid, thus accelerating the electrons. At $9000 \mathrm{~V}$, the electrons' velocity was approximately $0.18 \mathrm{c}$, where $c$ is the velocity of light. Fig. $5 b$ shows that the phase velocity ranged from $0.235 \mathrm{c}$ to $0.1 \mathrm{c}$, and the group velocity ranged from $0.18 \mathrm{c}$ to $0.14 \mathrm{c}$. For this normal dispersion system, the group velocity was equal to the energy velocity. Thus, the electrons achieved sufficient energy to obtain an effective deflection and the deflection sensitivity was $20 \mathrm{~mm} / \mathrm{KV}$.

\section{System simulation}

To validate this proposed traveling wave plate, we established a femtosecond SC model in CST software, as shown in Fig. 6. The model comprised a cathode, a grid electrode, a TWD, and a magnetic lens.

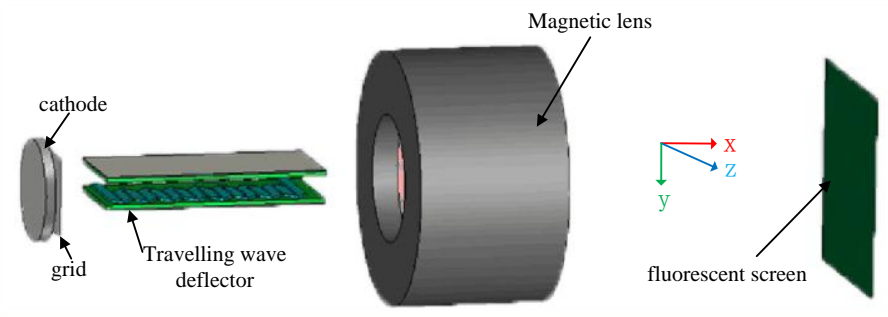

Fig. 6. Simulation model of femtosecond SC in CST 
Rayleigh criterion is a useful tool to evaluate the degree of distinction. Narrow electronic pulse sequences were emitted from the surface of the cathode, which had a constant time interval. The temporal resolution was simulated and calculated by those electronic pulse sequences, as shown in Fig. 7.

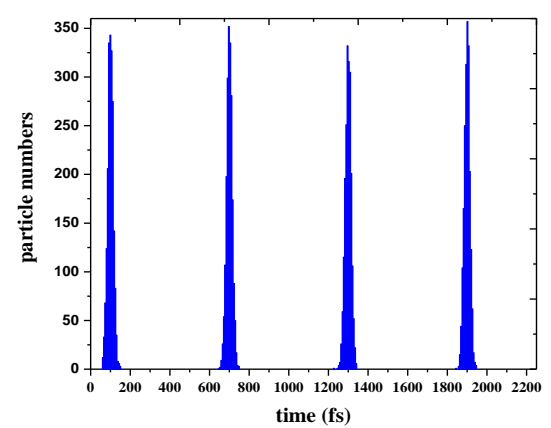

Fig. 7. Electronic pulse sequences

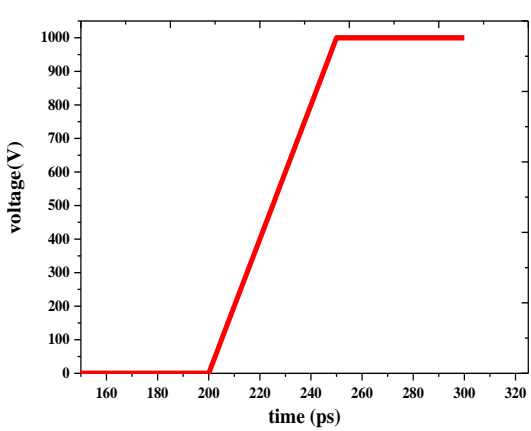

Fig. 8. Scanning signal

Four electronic pulse beams, with 2000 electrons adopted in each beam, were emitted from the cathode. The electrons obeyed the following principles: The initial energy obeyed the $\beta$ distribution ranging from 0 to $0.6 \mathrm{eV}$, the launching elevation obeyed the cosine distribution ranging from 0 to $\frac{\pi}{2}$, the azimuth obeyed the uniform distribution ranging from 0 to $2 \pi$, the time interval between 2 adjacent electronic pulses was $600 \mathrm{fs}$, and the full width at half maximum was $50 \mathrm{fs}$. To achieve dynamic scanning, a high-speed scanning signal as shown in Fig. 8 was imported into the TWD from port1. Fig. 8 shows that the scanning signal rose to $1000 \mathrm{~V}$ in only 50 ps linearly. However, we note that the electrons could achieve the largest deflection in the TWD when the electrons entered the TWD and the scanning signal was in a linear segment.

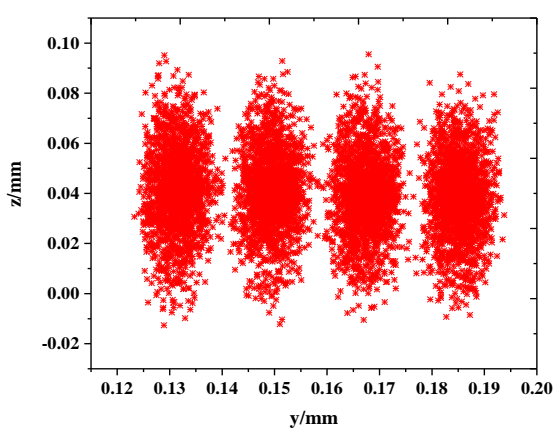

(a)

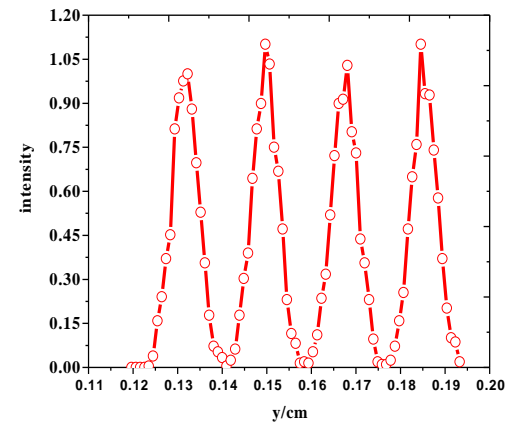

(b)

Fig. 9. Sweeping results of electron pulses with a duration of 600 fs. (a) Beam spot of electron pulses on the screen. (b) Intensity distribution of electrons in the meridian direction.

Fig. 9a shows the electron distribution as recorded on a CCD, and Fig. 9b shows the electron distribution curve in the meridian direction. As shown in Fig. 9b, the 4 electronic pulse beams continuous in time were transformed into spatial information. Therefore, this femtosecond streak tube can achieve a dynamic temporal resolution of less than $600 \mathrm{fs}$. 


\section{Experiments}

We did a system experiment to test the dynamic temporal resolution. As shown in Fig. 10a, the optical path was adopted.

149

150

151

152

153

154

155

156

157

158

159

160

161

162

163

164

165

166

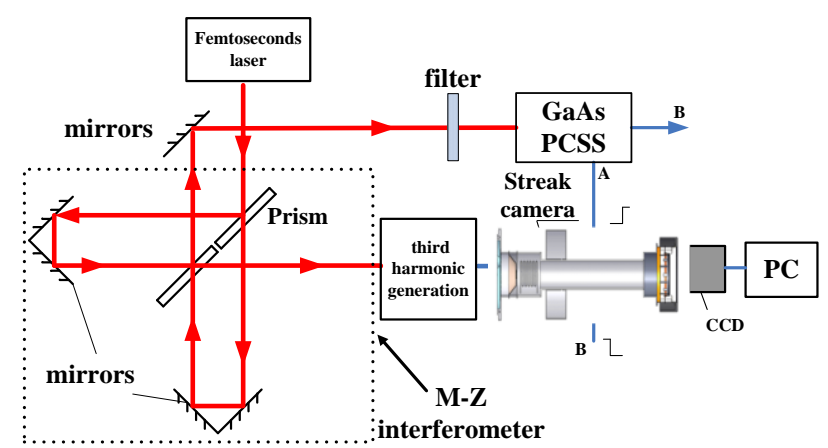

(a)

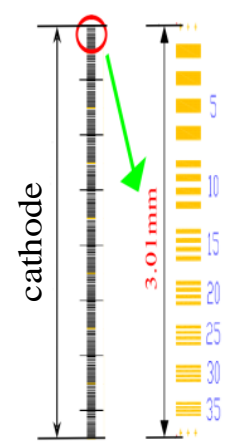

(b)

Fig. 10. System experiment. (a) Optical path.

(b) Adopted cathode.

A femtosecond laser pulse was emitted from a Ti sapphire laser whose central wavelength was $800 \mathrm{~nm}$. The laser pulse was divided into 2 beams by a beam splitter; the splitting ratio was 2:8. One beam was received by photoconductive semiconductor switches (PCSS) to generate a scanning signal; Two femtosecond laser pulses were generated when the other beam passed through a Mach-Zehnder interferometer, and the time interval of this two femtosecond laser pulses can be adjusted by a etalon, then it was focused by a lens where the focal distance was $40 \mathrm{~mm}$, and a third harmonic (wavelength $266 \mathrm{~nm}$ ) was generated due to the nonlinear effect. Finally, the third harmonic and the fundamental light were separated by a prism. The third harmonic was made incident on the Au cathode by a light-path adjustment. To ensure synchronization between the electron pulse and the optical pulse, the optical delay line was adopted. The streak images of two femtosecond laser pulses were recorded by electrons bombarding CCD (EBCCD). The Au photocathode was adopted in the experiment, as shown in Fig. 10b, and was split into several slits to test the spatial resolution; the result is reported in [8]. A mesh anode was placed downstream to face the Au-coated surface. The mesh anode generated a uniform high-gradient field of $18 \mathrm{MV} / \mathrm{m}$ between the photocathode and the anode.

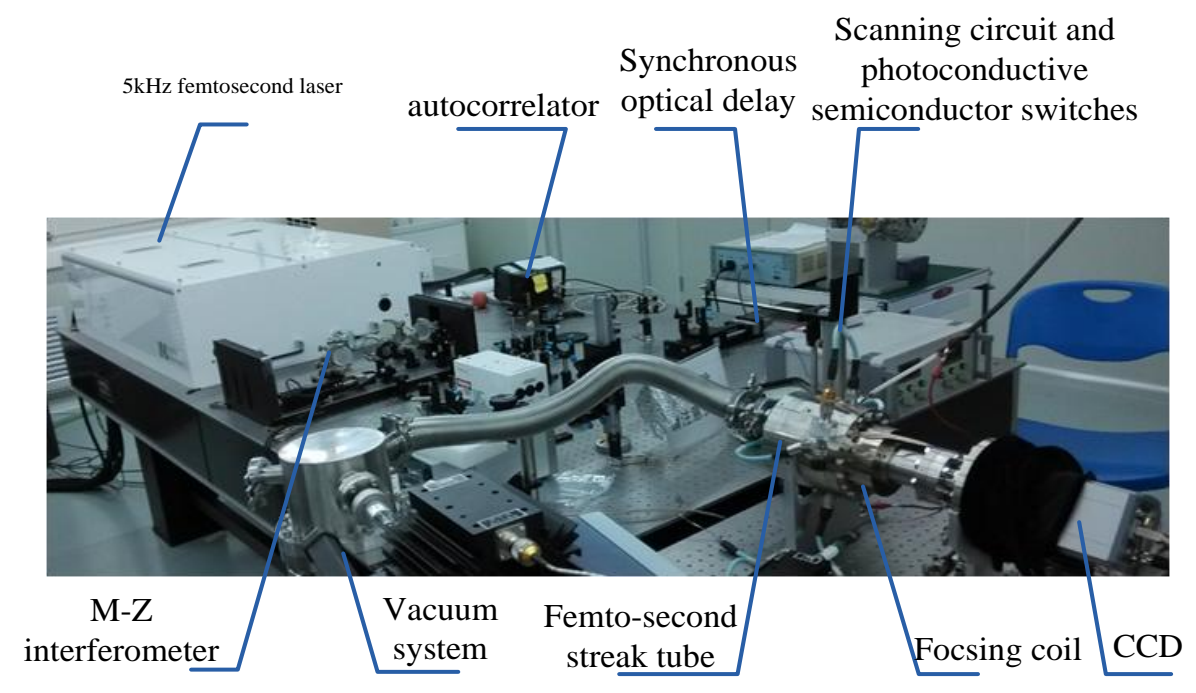

Fig. 11. System experiment setup 
The system experiment setup is shown in Fig. 11. The synchronous optical delay model was used to adjust the streak images into a proper position, and the vacuum system ensured an efficient degree of vacuum. The pulse width of the femtosecond laser was measured by an autocorrelator at approximately $70 \mathrm{fs}$. The voltage applied to the cathode was $9000 \mathrm{~V}$, the direct bias voltage on the TWD was $425 \mathrm{~V}$, the scanning signal that was generated by photoconductive semiconductor switches rose to $1500 \mathrm{~V}$ within $50 \mathrm{ps}$, the focusing current was $0.317 \mathrm{~A}$, and the power of the injected laser was $170 \mathrm{~mW}$. In addition, the jitter of the photoconductor switcher was also measured by the method in [9]; it was approximately 287 fs. Figures $12 \mathrm{a}$ and $12 \mathrm{c}$ are the streak images when the time interval was $800 \mathrm{fs}$ and $900 \mathrm{fs}$ respectively when the repetitive frequency was $2 \mathrm{kHz}$ and the integration time of CCD was 500 milliseconds.

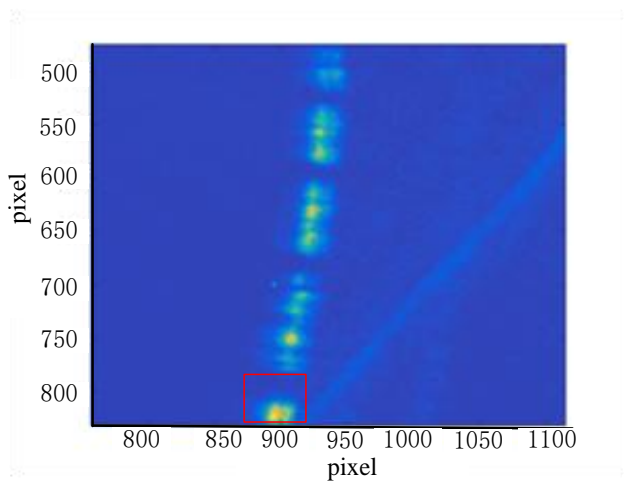

(a)

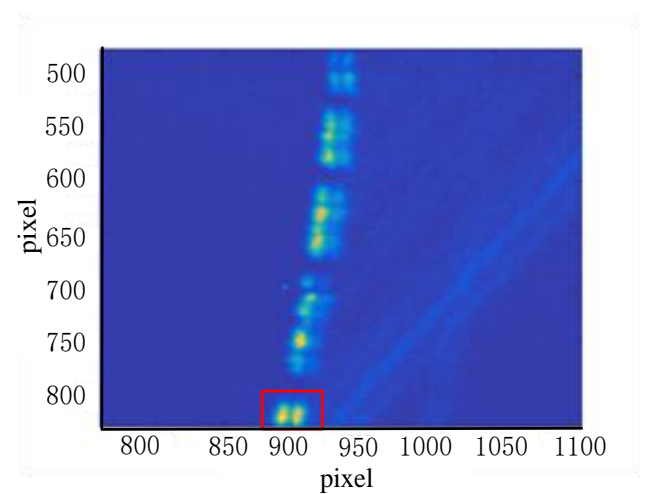

(c)

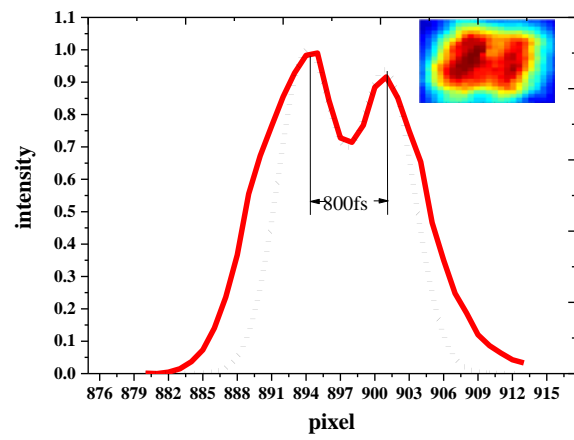

(b)

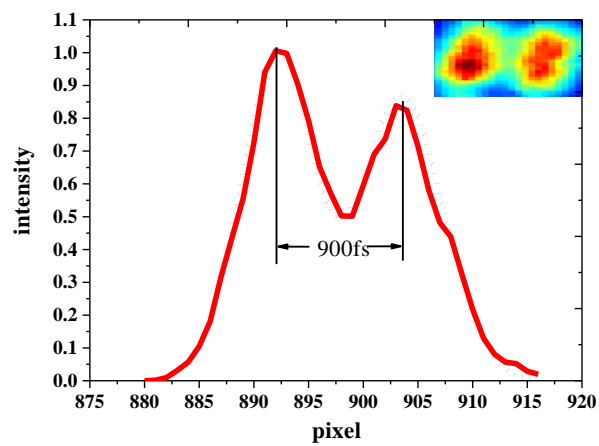

(d)

Fig. 12. Streak images and intensity distributions. (a) Streak image at $800 \mathrm{fs}$. (b) Intensity distribution of electrons in the meridian direction. (c) Streak image at 900 fs. (d) Intensity distribution of electrons in the meridian direction.

Fig. 12a was the streak image of two femtosecond laser pulses when the time interval was 800fs and Fig. 12c was the streak image of two femtosecond laser pulses when the time interval was 900fs. Figs. 12b and 12d are temporal resolutions defined by Rayleigh's criterion. The dotted lines are Gaussian fits of the experimental results, which showed that the camera can achieve a dynamic temporal resolution of less than $800 \mathrm{fs}$.

\section{Conclusions}

In this study, a new TWD based on a microstrip line was proposed, and its dispersion and 
pass width were simulated. A system simulation for dynamic temporal resolution was also done; the result showed that the dynamic temporal resolution was less than $600 \mathrm{fs}$. In addition, the femtosecond SC was subjected to a series of experiments. The dynamic temporal resolution was tested and the results showed an excellent resolution of $900 \mathrm{fs}$. In following work, we will optimize the electron optics system and the TWD to achieve higher dynamic temporal resolution by developing photoconductive semiconductor switches with higher stability and optimizing the cathode voltage to get greater deflection sensitivity. Chang designed the SC with a 280fs temporal resolution when the jitter of the photoconductor switcher was only $30 \mathrm{fs}$. However, the jitter in our system was $287 \mathrm{fs}$. Once the jitter was reduced, the temporal resolution would be improved. In this way, a femtosecond SC can obtain higher temporal resolution to meet the demands of ICF and other diagnostic uses.

\section{Acknowledgment}

This work was supported by National Science Foundation of China (51271140, 61205022).

\section{References}

[1] Lindl, J. D., Amendt, P., Berger, R. L., Glendinning, S. G., Glenzer, S. H., Haan, S. W., Kauffman, R. L., Landen, O. L., and Suter, L. J, Phys. Plasmas 11, 339 (2004).

[2] Glenzer, S. H., Callahan, D. A., MacKinnon, A. J., Kline, J. L., Grim, G., et al, Phys. Plasmas 19, 056318 (2012).

[3] Z. Chang, A. Rundquist, J. Zhou, M. M. Murnane, H. C. Kapteyn, X. Liu,B. Shan, J. Liu, M. Gong, and X. Zhang, Phys. Lett. 69,133(1996).

[4] V. P. Degtyareva, V. S. Belolipetski, G. I. Bryukhnevich, S. R. Ivanova, G. P.Levina, V. A. Makushina, N. D. Polikarkina, Z. M. Semichastnova, M.Ya. Schelev, SPIE, 4948:281-290 (2003).

[5] Takeshita T, Suzuki K, Kinoshita K, et, al, SPIE, 4183: 266-262(2001).

[6] Jain P K, Basu B N, IEEE Traps Electron Devices, 34(12): 2643-2648 (1987).

[7] Huang G, Byrd J M, Feng Jun, et al, Proceedings of EPAC, 101: 1250-1252 (2006).

[8] Liu R, Tian J S, Li H, Wang Q Q, Wang C, Wen W L, Lu Y, Liu H L, Cao X B, Wang J F, Xu X Y, Wang X, Acta Phys. Sin. 63 (058501) (2014) (in Chinese).

[9] J. Liu, J. Wang, B. Shan, C. Wang, and Z. Chang, Proc. SPIE, vol. 5194, pp. 123-126, Jan. (2004).

[10] Zhu Min, Tian Jin Shou, Wen Wen Long, Wang Jun Feng, Cao Xi Bin, Lu Yu, Xu Xiang Yan, Sai Xiao Feng, Liu Hu Lin, Wang Xing, Li Wei Hua, Acta Phys. Sin. 64 (9): 1-8 (2015) (in Chinese). 